Draft VERSION JUNE 6, 2022

Typeset using $\mathrm{LAT}_{\mathrm{E}} \mathrm{X}$ twocolumn style in AASTeX62

\title{
THE TRANSITION FROM A LOGNORMAL TO A POWER-LAW COLUMN DENSITY DISTRIBUTION IN MOLECULAR CLOUDS: AN IMPRINT OF THE INITIAL MAGNETIC FIELD AND TURBULENCE
}

\author{
Sayantan Auddy, ${ }^{1}$ Shantanu Basu, ${ }^{2}$ And Takahiro Kudoh ${ }^{3}$ \\ ${ }^{1}$ Institute of Astronomy and Astrophysics, Academia Sinica, Taipei 10617, Taiwan \\ ${ }^{2}$ Department of Physics and Astronomy, The University of Western Ontario, London, ON N6A 3K7, Canada \\ ${ }^{3}$ Faculty of Education, Nagasaki University, 1-14 Bunkyo-machi, Nagasaki 852-8521, Japan
}

\begin{abstract}
We introduce a theory for the development of a transitional column density $\Sigma_{\mathrm{TP}}$ between the lognormal and the power-law forms of the probability distribution function (PDF) in a molecular cloud. Our turbulent magnetohydrodynamic simulations show that the value of $\Sigma_{\mathrm{TP}}$ increases as the strength of both the initial magnetic field and turbulence increases. We develop an analytic expression for $\Sigma_{\mathrm{TP}}$ based on the interplay of turbulence, a (strong) magnetic field, and gravity. The transition value $\Sigma_{\mathrm{TP}}$ scales with $\mathcal{M}_{0}^{2}$, the square of the initial sonic Mach number, and $\beta_{0}$, the initial ratio of gas pressure to magnetic pressure. We fit the variation of $\Sigma_{\mathrm{TP}}$ among different model clouds as a function of $\mathcal{M}_{0}^{2} \beta_{0}$, or equivalently the square of the initial Alfvénic Mach number $\mathcal{M}_{\mathrm{A} 0}^{2}$. This implies that the transition value $\Sigma_{\mathrm{TP}}$ is an imprint of cloud initial conditions and is set by turbulent compression of a magnetic cloud. Physically, the value of $\Sigma_{\mathrm{TP}}$ denotes the boundary above which the mass-to-flux ratio becomes supercritical and gravity drives the evolution.
\end{abstract}

Keywords: ISM: clouds - magnetic fields - magnetohydrodynamics (MHD) — stars: formation

\section{INTRODUCTION}

The column density probability distribution function (PDF) provides an effective way to analyze the dynamics and the evolution of molecular clouds from both observational (e.g., Burkhart et al. 2015b; Schneider et al. 2015a, 2016; Pokhrel et al. 2016) and theoretical (e.g., Burkhart 2018; Körtgen et al. 2019) perspectives. Numerical simulations have established that nonself-gravitating gas with driven turbulence results in many interacting shocks that yield a lognormal density or column density PDF (e.g., Padoan et al. 1997; Passot \& Vázquez-Semadeni 1998; Scalo et al. 1998; Federrath et al. 2008; Molina et al. 2012). The addition of self-gravity into simulations introduces a highdensity power-law tail to the PDF (Kritsuk et al. 2011; Ballesteros-Paredes et al. 2011; Collins et al. 2012; Federrath \& Klessen 2013; Ward et al. 2014; Auddy et al. 2018). Observed column density PDFs have an underlying lognormal shape with an additional power-law tail (Kainulainen et al. 2009; Alves et al. 2014) that starts at a transitional column density (Schneider et al. 2015b).

Corresponding author: Sayantan Auddy sauddy@asiaa.sinica.edu.tw
A lognormal shape is associated with quiescent clouds that do not have active star formation (Kainulainen et al. 2009; Lombardi et al. 2015; Schneider et al. 2015b). In contrast, the active star-forming clouds have an excess of high column density with a prominent power-law tail as well as a lognormal peak. The lognormal feature is often considered to be a direct imprint of driven supersonic turbulence and its width is attributed to the strength of the sonic Mach number (Collins et al. 2012; Molina et al. 2012; Burkhart et al. 2015a). However, it has also been suggested that the lognormal may be a more general characteristic that is set by both supersonic turbulence and gravitationally-driven ambipolar diffusion (Tassis et al. 2010) or global gravitational contraction (Ballesteros-Paredes et al. 2011).

The power-law part of the PDF (Schneider et al. 2013; Alves et al. 2017) is a signature of gravitational contraction (due to the self-gravity of the gas) and can be associated with the formation of condensed cores. The PDF $d N / d \log \Sigma \propto \Sigma^{-\alpha}$ has an index $\alpha=2$ in the limit of isothermal gravitational contraction (see e.g., Appendix A of Auddy et al. 2018), and is set by the density profiles within dense cores. However, the observed $\alpha$ is sometimes steeper. For instance, molecular clouds like Polaris and Pipe have power-law indices $\alpha=3.9$ and $\alpha=3.0$, 
respectively (Lombardi et al. 2015). These clouds are diffuse and have much less star formation compared to active star-forming clouds like Aquila which have $\alpha \approx 2$ (Könyves et al. 2015). Auddy et al. (2018) showed that the magnetic field can significantly affect the slope of the power-law tail. Clouds with a strong magnetic field (subcritical mass-to-flux ratio) and small amplitude initial perturbations develop a steep power-law tail $(\alpha \approx 4)$, consistent with gravitationally-driven ambipolar diffusion leading to shallower core density profiles than in a hydrodynamic collapse. In contrast, turbulent subcritical clouds retain the lognormal shape for a long time and eventually develop a power-law tail with $\alpha \approx 2$ in a region that has become supercritical due to turbulence-enhanced ambipolar diffusion.

Each PDF has at least three measurable parameters: the width of the lognormal part, the slope of the powerlaw tail, and the transitional column density $\Sigma_{\mathrm{TP}}$ that separates the lognormal from the power-law portion. Many theoretical studies have associated the standard deviation $\sigma$ of the lognormal distribution with the sonic Mach number of driven turbulence (e.g., Federrath et al. 2008; Molina et al. 2012). The power-law tail develops when self-gravity is introduced into a driven turbulence simulation (Collins et al. 2012; Federrath \& Klessen 2013). Decaying hydrodynamic turbulence simulations with self-gravity also show a rapid development of a power-law tail in the PDF (Kritsuk et al. 2011; Ballesteros-Paredes et al. 2011; Ward et al. 2014). Previous studies have not developed a theory for the location of $\Sigma_{\mathrm{TP}}$, although Burkhart et al. (2017) and Imara \& Burkhart (2016) have proposed that it is associated with the $\mathrm{H}$ I-to- $\mathrm{H}_{2}$ transition in the interstellar medium. This has some appeal since H I clouds are known to be nonself-gravitating whereas molecular clouds exist at higher pressures and are considered to be self-gravitating (Blitz 1991). However, observations of many molecular clouds show that the transition occurs within the molecular gas, and that the value of $\Sigma_{\mathrm{TP}}$ is unique to each cloud, e.g., NGC 3603, Carina, Maddalena, and Auriga all have different deviation points (DP) as listed in Table 1 of Schneider et al. (2015b). This implies that $\Sigma_{\text {TP }}$ is an imprint of initial conditions inherent to a particular starforming cloud and is set by physical processes. Furthermore, the longstanding well-known low efficiency of star formation (e.g., Goldsmith et al. 2008) within molecular clouds means that most of the molecular gas mass is not within gravitationally-contracting dense cores that account for the power-law portion of the PDF.

In this Letter, we focus on the physical origin of the transition point $\Sigma_{\mathrm{TP}}$, using a different approach than adopted in most previous studies of the PDF. Auddy et al. (2018) showed that in a decaying turbulence scenario with supercritical mass-to-flux ratio, the lognormal body is quickly lost and a power law is developed for essentially all densities past the peak. Simulations with constant turbulent driving with Fourier space perturbations are able to maintain a distinct lognormal body and a power law tail in the PDF, although that may be an artefact of turning on self-gravity only after a steady turbulent driving has been established. Since real molecular clouds do not have a "switch-on" gravity, we investigate here the scenario that a strong magnetic field (i.e., subcritical mass-to-flux ratio) supports large amplitude oscillations (even while there is an overall decay of turbulence) that result in the maintenance of a lognormallike body of the PDF. Gravity is always at work, but can only win out in dense regions that have undergone a rapid turbulence-accelerated ambipolar diffusion. In this case, Auddy et al. (2018) showed that a power-law tail with $\alpha \approx 2$ is added to the lognormal-like body of the PDF. Here, we develop a self-consistent theory of the origin of $\Sigma_{\mathrm{TP}}$ in this scenario, and test it against a suite of simulations with different initial conditions. We find a direct link between $\Sigma_{\mathrm{TP}}$ and the relative importance of turbulence and magnetic fields in the initial cloud. In Section 2, we present numerical simulations that study the properties of the column density PDFs. In Section 3 we derive an analytic expression for $\Sigma_{\mathrm{TP}}$ based on a model of turbulent compression of a magnetized cloud, and compare with the numerical results. In Sections 4 and 5 we discuss and summarize our results, respectively.

\section{COLUMN DENSITY PDFS}

We study the time evolution of the column density PDFs for five different models using three-dimensional magnetohydrodynamic (MHD) simulations including self-gravity and ambipolar diffusion. The numerical setup is similar to the ones previously used in Kudoh et al. (2007); Kudoh \& Basu (2011) and Auddy et al. (2018), and we run with a number of grid points in each direction $\left(N_{x}, N_{y}, N_{z}\right)=(512,512,20)$.

We consider models with a subcritical initial mass-toflux ratio so that the magnetic field strength is dynamically important. The initial turbulent flow field causes the PDF to have a predominantly lognormal shape. However, as the cloud evolves, it forms compressed regions due to the large-scale flow and develops pockets of high column density. It then rebounds and shows oscillations. With each successive compression, more regions with high column density develop and cause a gradual widening of the width of the lognormal, even though 

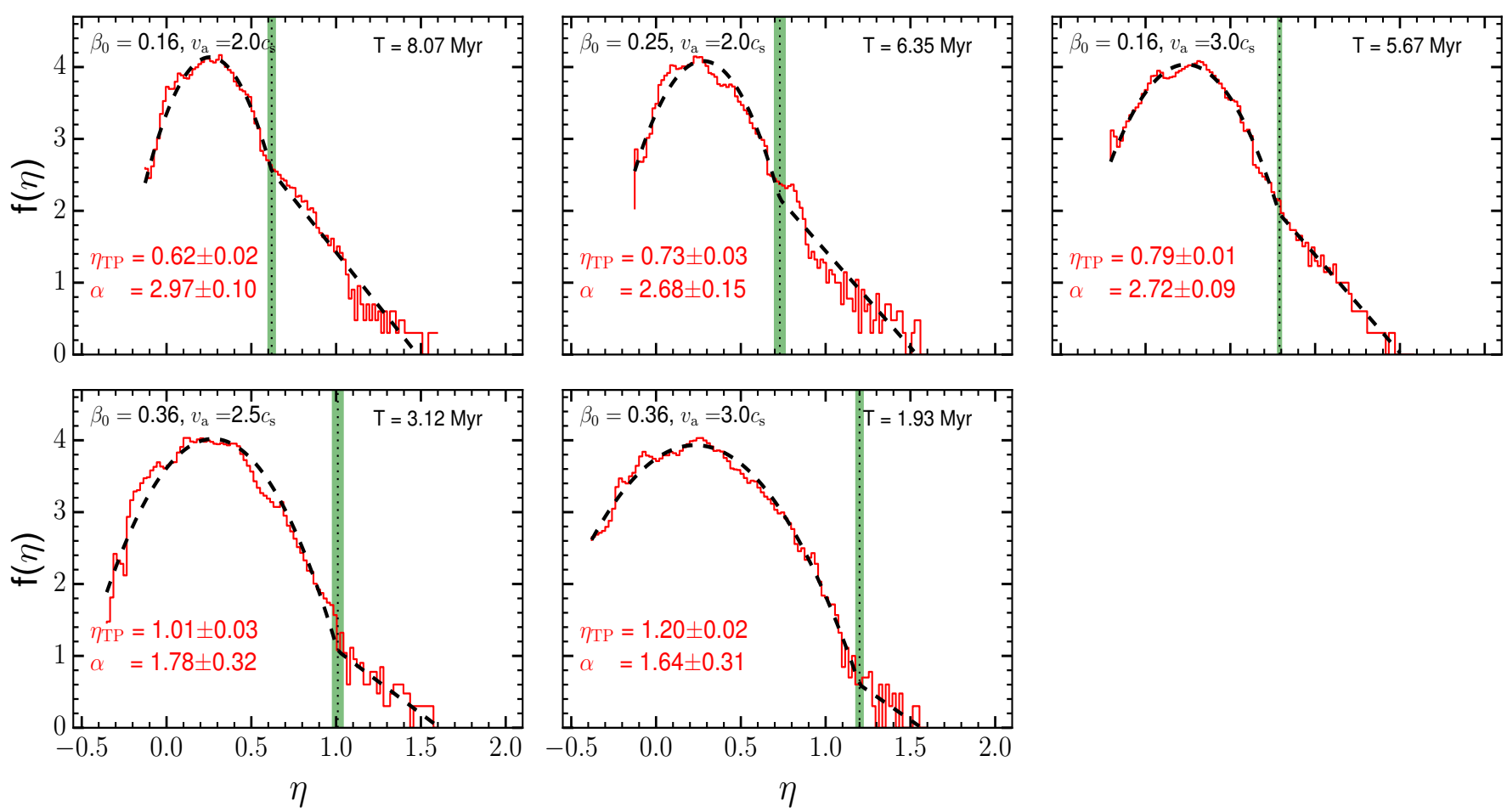

Figure 1. The column density PDFs of simulated models of molecular clouds with different initial conditions. The initial plasma $\beta_{0}$ and the turbulence amplitude $v_{\mathrm{a}}$ is specified on the top left of each panel. Each column density PDF is fitted with Equation (3). The vertical black dotted line marks the logarithmic column density value (i.e., transition point $\eta_{\mathrm{TP}}$ ) at which the power-law begins. The green shaded region represents the standard deviation of the transitional column density value. The best-fit parameters $\left(\alpha, \eta_{\mathrm{TP}}\right)$ are obtained using the MCMC method in python.

the turbulence is decaying (see also Ward et al. (2014); Tassis et al. (2010); Auddy et al. (2018)).

We follow the time evolution of the column density PDF for each model with different initial conditions. The PDF evolves over time from primarily having a lognormal shape at early times to developing a power-law tail (final time $\sim 1 \mathrm{Myr}$ ) when gravity dominates. The power-law develops after several oscillations, as the local pockets of higher column density become supercritical and go into a runaway collapse.

\subsection{Numerical Parameters}

The initial state has a uniform density in $x, y$ and is stratified in the $z$-direction with a scale length $H_{0}=$ $c_{\mathrm{s} 0} / \sqrt{2 \pi G \rho_{0}}$, where $c_{\mathrm{s} 0}$ and $\rho_{0}$ are the isothermal sound speed and density at the midplane $z=0$. For more details of the initial setup, see Kudoh et al. (2007); Kudoh $\&$ Basu (2011); Auddy et al. (2018). We choose $H_{0}, c_{\mathrm{s} 0}$ and $\rho_{0}$ as units of length, velocity and density respectively. This gives the unit of time $t_{0} \equiv H_{0} / c_{\mathrm{s} 0}$. The ratio of the initial gas to magnetic pressure at $z=0$ is

$$
\beta_{0}=\frac{8 \pi c_{\mathrm{s} 0}^{2} \rho_{0}}{B_{0}^{2}}
$$

where $B_{0}$ is the initially uniform vertical magnetic field. We input Gaussian random velocity fluctuations of amplitude $v_{a}$ for each of the $x$ - and $y$-components of velocity, in which the Fourier spectrum is $v_{k}^{2} \propto k^{-4}$. Appropriate choices of $\rho_{0}$ and $c_{s 0}$ lead to dimensional values of standard quantities. For example, if $n_{0} \equiv$ $\rho_{0} / m_{n}=10^{4} \mathrm{~cm}^{-3}$ where $m_{n}=2.33 \times 1.67 \times 10^{-24}$ $\mathrm{g}$, and $c_{\mathrm{s} 0}=0.2 \mathrm{~km} \mathrm{~s}^{-1}$, we get $H_{0} \simeq 0.05 \mathrm{pc}$ and $t_{0} \simeq 2.5 \times 10^{5}$ yrs. If $\beta_{0}=0.16$, Equation (1) yields $B_{0} \simeq 50 \mu \mathrm{G}$. The initial column density is $\Sigma_{0}=\rho_{0} H_{0} \simeq$ $6 \times 10^{-3} \mathrm{~g} \mathrm{~cm}^{-2}$, therefore the number column density is $N_{0} \equiv \Sigma_{0} / m_{n} \simeq 1.5 \times 10^{21} \mathrm{~cm}^{-2}$.

\subsection{Fitting Functions}

In order to characterise the shape of the PDFs including the transition from the lognormal to power-law tail we consider two fitting functions: a purely lognormal function and a piecewise function that is a combination of a lognormal and a power law. If $f(\eta)$ is the PDF, the lognormal model is

$$
f(\eta)_{\mathrm{LN}}=\log \left[A \frac{1}{\sqrt{2 \pi \sigma^{2}}} \exp \left(-\frac{(2.3 \eta-\mu)^{2}}{2 \sigma^{2}}\right)\right]
$$


Table 1. Model and Fit Parameters

\begin{tabular}{llllcccc}
\hline \hline Model & $v_{\mathrm{a}} / c_{\mathrm{s}}$ & $\beta_{0}$ & $\mathcal{M}_{0}^{2} \beta_{0}$ & $\eta_{\mathrm{TP}}$ & $|\alpha|$ & $\mu$ & $\sigma$ \\
\hline $\mathrm{T} 1$ & 2.0 & 0.16 & 1.28 & $0.62 \pm 0.02$ & $3.0 \pm 0.1$ & $0.59 \pm 0.01$ & $0.31 \pm 0.01$ \\
$\mathrm{~T} 2$ & 2.0 & 0.25 & 2.00 & $0.74 \pm 0.03$ & $2.7 \pm 0.2$ & $0.64 \pm 0.02$ & $0.35 \pm 0.01$ \\
$\mathrm{~T} 3$ & 3.0 & 0.16 & 2.88 & $0.79 \pm 0.02$ & $2.7 \pm 0.1$ & $0.55 \pm 0.01$ & $0.41 \pm 0.01$ \\
$\mathrm{~T} 4$ & 2.5 & 0.36 & 4.50 & $1.01 \pm 0.03$ & $1.8 \pm 0.3$ & $0.63 \pm 0.01$ & $0.46 \pm 0.01$ \\
$\mathrm{~T} 5$ & 3.0 & 0.36 & 6.48 & $1.20 \pm 0.02$ & $1.6 \pm 0.3$ & $0.53 \pm 0.01$ & $0.57 \pm 0.01$ \\
\hline
\end{tabular}

NoтE-Fit parameters for the piecewise lognormal and power-law function are $\alpha, \sigma, \mu$, and $\eta_{\mathrm{TP}}$. $\beta_{0}$ is the initial ratio of thermal to magnetic pressure at $z=0, v_{a}$ is the amplitude of the initial velocity fluctuation and $\mathcal{M}_{0}$ is the sonic Mach number.

where $\eta=\log \left(\Sigma / \Sigma_{0}\right), \quad A=\ln (10) \times N_{\text {total }}^{1} \times$ $\Delta \log \left(\Sigma / \Sigma_{0}\right)$ is the normalization constant, $\mu$ is the mean and $\sigma$ is the standard deviation. The data is binned with a uniform spacing of $\Delta \log \left(\Sigma / \Sigma_{0}\right) \simeq 0.02$. For the piecewise function (see also Myers 2015; Pokhrel et al. 2016) we consider a combination of a lognormal and a power law:

$$
\begin{aligned}
f(\eta)_{\mathrm{LNPL}} & =\quad f(\eta)_{\mathrm{LN}}, \quad \text { if } \eta \leq \eta_{\mathrm{TP}} \\
& =f\left(\eta_{\mathrm{TP}}\right)_{\mathrm{LN}}+\alpha \eta, \text { if } \eta>\eta_{\mathrm{TP}}
\end{aligned}
$$

where $\alpha$ is the index of the power law and $\eta_{\mathrm{TP}} \equiv$ $\log \left(\Sigma_{\mathrm{TP}} / \Sigma_{0)}\right)$ is the logarithmic value of the transition column density. Thus, we have two fitting functions and four free parameters: $\mu, \sigma, \eta_{\mathrm{TP}}$, and $\alpha$.

We use this piecewise four-parameter function rather than a mathematically simpler three-parameter continuous function, for example the modified lognormal power law distribution (Basu et al. 2015), since it clearly identifies a transition point $\eta_{\mathrm{TP}}$. We are then also using the same means to identify the transition point as used in observational analyses like Pokhrel et al. (2016).

\subsection{Fitting the Simulation Data}

The value of the fit parameters is essential to characterise the shape of the column density PDFs. However, the column density PDFs are evolving in time with their shape changing from purely lognormal to a hybrid function. Thus it is essential to have a robust fitter that can capture the transition and identify $\eta_{\mathrm{TP}}$. We fit the column density PDFs at different times using $f(\eta)_{\mathrm{LN}}$ (Equation 2) and $f(\eta)_{\text {LNPL }}$ (Equation 3) and compute the resulting $\chi^{2}$ values. We accept $f(\eta)_{\text {LNPL }}$ only when its $\chi^{2}$ value is less than $20 \%$ of that of the simpler lognormal function $f(\eta)_{\mathrm{LN}}$ and the power-law index $\alpha<5$. For models that are fit with $f(\eta)_{\mathrm{LNPL}}$, we further use a Markov Chain Monte Carlo (MCMC) method (van Dyk 2003) as a second fitter. This gives us more robust best-fitting values from the parameter space along

\footnotetext{
1 Total length of the sample space of $\eta$ from the simulations, i.e., $512 \times 512$.
}

with reliable uncertainties. We have used the PYTHON package Pymc for this purpose (Patil et al. 2010).

The power-law tail appears during the final stages of the simulations, primarily due to ambipolar-diffusiondriven gravitational contraction. All the free parameters evolve moderately, including the transitional column density $\eta_{\mathrm{TP}}$, which grows by $\approx 10 \%$ from its initial appearance until the final time step. For simplicity we only consider the column density PDFs at the end of the simulation when the maximum density has reached $100 \rho_{0}$. Runaway collapse has ensued in the high density regions at this time. While we cannot follow the PDFs into the protostellar phase in these simulations, we anticipate that the large-scale maps of the PDFs will remain largely the same.

Figure 1 shows the column density PDFs of five models with different initial conditions (i.e., $\beta_{0}$ and $v_{\mathrm{t} 0}$ ) along with the best fit lognormal and power-law functions. The results are summarized in Table 1 . The run time for each simulation is indicated on the top right of each plot. The best-fitting parameters $\alpha$, and $\eta_{\mathrm{TP}}$ are also shown. The plots are arranged from top left to bottom right according to increasing values of $\mathcal{M}_{0}^{2} \beta_{0}$. The black dotted line marks the column density value at which the lognormal PDF ends and the power-law tail begins. The green shaded region shows the standard deviation of the $\eta_{\mathrm{TP}}$ value obtained from the MCMC fit.

The transition point $\eta_{\mathrm{TP}}$ shifts toward higher column density with increasing value of $\mathcal{M}_{0}^{2} \beta_{0}$. For example, it is minimum for model $\mathrm{T} 1\left(\mathcal{M}_{0}^{2} \beta_{0}=1.28\right)$ and maximum for model T5 $\left(\mathcal{M}_{0}^{2} \beta_{0}=6.48\right)$ with $\eta_{\mathrm{TP}}=0.62 \pm 0.02$ and $\eta_{\mathrm{TP}}=1.20 \pm 0.02$, respectively. The value depends on the strength of the initial magnetic field and the amplitude of the velocity perturbation.

\section{ANALYTIC MODEL}

We can understand the physical origin of the transition point with an analytic model in which the magnetic field is dynamically important. The cloud flattens along the mean magnetic field direction $(z)$ and the subsequent evolution is primarily perpendicular to the magnetic field. The mass-to-flux ratio is subcritical un- 
til ambipolar diffusion creates supercritical pockets that are prone to collapse. Turbulence causes the creation of locally compressed regions that have a pressure balance between magnetic and ram pressure. This results in the formation of magnetic ribbons (Auddy et al. 2016, see also Kudoh \& Basu (2014)). The cloud is stratified along the $z$-direction with compression along the $x-y$ plane. We simplify the analysis and assume that the thermal pressure is negligible compared to the magnetic pressure and the ram pressure of the flow. The pressure due to the magnetic field $B$ upon compression balances the initial pressure due to the background magnetic field $B_{0}$ and the external ram pressure in the $x-y$ direction:

$$
H \frac{B^{2}}{8 \pi}=H_{0}\left(\rho_{0} v_{\mathrm{t} 0}^{2}+\frac{B_{0}^{2}}{8 \pi}\right)
$$

where $v_{\mathrm{t} 0}=\sqrt{2} v_{a}$ is the nonlinear flow speed. This results in a quasi-equilibrium state as compression ceases and oscillations begin. The gas has already settled into a hydrostatic equilibrium along the $z$ - direction and the cloud has a half-thickness

$$
H=\frac{c_{\mathrm{s}}}{\sqrt{2 \pi G \rho}}
$$

(Spitzer 1942). Integrating the density along the scale height $H$ in the $z$-direction gives the column density

$$
\Sigma=2 \rho H .
$$

The initial density compression is very high (see figure 1 in Kudoh \& Basu 2008) but the subcritical mass-toflux ratio results in a strong rebound. We consider that the cloud is nearly flux frozen during its initial compression, i.e., $B / \Sigma=$ constant, as the ambipolar diffusion time is much longer than the compression time. Using (5) and (6) in Equation (4) along with the flux frozen condition we find

$$
\frac{\Sigma}{\Sigma_{0}}=\left[v_{\mathrm{t} 0}^{2}\left(\frac{8 \pi \rho_{0}}{B_{0}^{2}}\right)+1\right]
$$

The force balance of Equation (7) gives a critical column density that we denote as $\Sigma_{\text {TP }}$. However, subsequent oscillations are not as strong due to decay of the initial turbulence amplitude $v_{\mathrm{t} 0}$ and loss of magnetic flux due to ambipolar diffusion. Allowing for such variations we rewrite Equation (7) in terms of the sonic Mach number $\mathcal{M}_{0}=v_{\mathrm{t} 0} / c_{\mathrm{s}}$, and plasma $\beta_{0}$ as

$$
\frac{\Sigma_{\mathrm{TP}}}{\Sigma_{0}}=a\left(\mathcal{M}_{0}^{2} \beta_{0}+1\right)
$$

where $a$ is a correction factor of order unity that contains uncertainties about the flux loss and turbulent decay.

Here $\mathcal{M}_{0}^{2} \beta_{0} \equiv 2 \mathcal{M}_{\mathrm{A} 0}^{2}$, where $\mathcal{M}_{\mathrm{A} 0}=v_{\mathrm{t} 0} / v_{\mathrm{A} 0}$ is the initial Alfvénic Mach number and $v_{\mathrm{A} 0}=B_{0} / \sqrt{\left(4 \pi \rho_{0}\right)}$ is the initial Alfvén speed in the midplane.

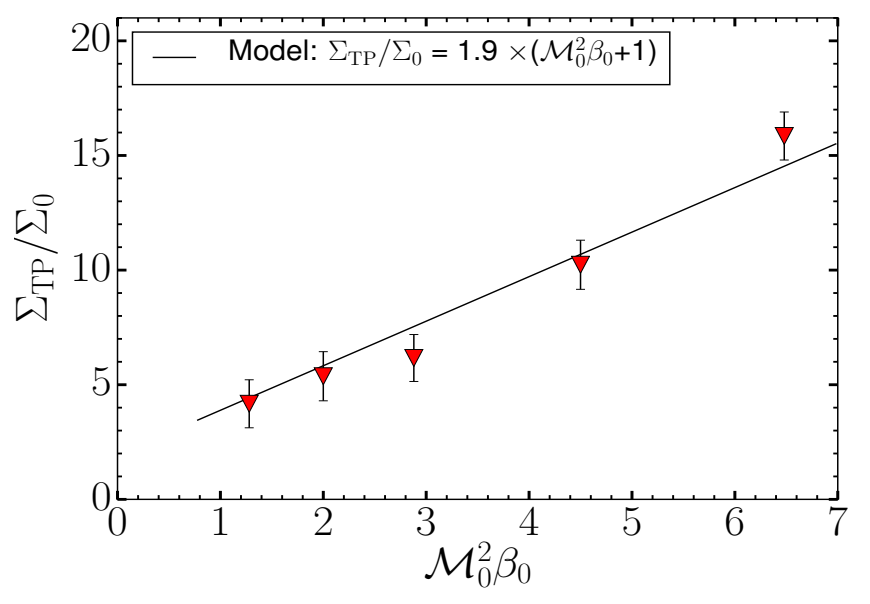

Figure 2. The normalized transition column density $\left(\Sigma_{\mathrm{TP}} / \Sigma_{0}\right)=10^{\eta_{\mathrm{TP}}}$ for different values of initial $\mathcal{M}_{0}^{2} \beta_{0}$ obtained from the simulations. The black line is the theoretical model (Equation 8) with the best fit value of $a=1.9$.

\subsection{Physical Interpretation of $\Sigma_{\mathrm{TP}}$}

Figure 2 shows the variation of the normalized transitional column density $\left(\Sigma_{\mathrm{TP}} / \Sigma_{0}\right)$ for simulated models with different initial values of $\mathcal{M}_{0}^{2} \beta_{0}$. We fit the analytic expression (Equation 8) to the simulation data and get a best fit value of $a=1.9$. We find a good agreement between the simulation data and our analytic model.

The transition from the lognormal to the power-law tail signifies both structural and morphological changes. It marks a transition from the ambient subcritical turbulent background $\left(\Sigma<\Sigma_{\mathrm{TP}}\right)$ to a compressed denser region where $\Sigma>\Sigma_{\mathrm{TP}}$. Due to ambipolar diffusion (ionneutral drift) the force balance between the ram pressure and the magnetic field gradually relaxes. There is a gradual loss of magnetic flux as the neutrals diffuse past the ions with each successive oscillation. The density is enhanced after each compression resulting in an increase of mass-to-flux ratio. The transitional column density $\Sigma_{\text {TP }}$ defines this cutoff beyond which the mass-to-flux ratio becomes critical. For $\Sigma>\Sigma_{\text {TP }}$ gravity becomes increasingly important and the power-law tail emerges.

Furthermore, with increased strength of the Alfvénic Mach number $\mathcal{M}_{\mathrm{A} 0}$ the initial compression is much stronger and it results in a higher density. This causes $\Sigma_{\mathrm{TP}}$ to shift towards higher values with increasing strength of $2 \mathcal{M}_{\mathrm{A} 0}^{2}$.

\section{DISCUSSION}

The shape of the column density PDF is an imprint of the initial conditions in a star-forming molecular cloud. It holds the key to finding the link between the structural properties and the ambient conditions that trigger 
star formation in molecular clouds. However, it is difficult to detect low column density material (the lognormal part) using dust emission and extinction measurement because of observational biases (Lombardi et al. 2015). The lognormal peak is sometimes considered an artefact arising due to data incompleteness (Alves et al. 2017) or undetectable due to insufficient sampling or limited field of view (Körtgen et al. 2019). The more robust observational quantities are the characteristic break $\Sigma_{\mathrm{TP}}$ in the PDF and the power-law slope $(\alpha)$, as these are less affected by such constraints (Lombardi et al. 2015).

Our model shows that $\Sigma_{\mathrm{TP}}$ is a measure of the initial Alfvénic Mach number $\mathcal{M}_{\mathrm{A} 0}$. Observations of $\Sigma_{\mathrm{TP}}$ can be used to infer the value of $\mathcal{M}_{\mathrm{A} 0} \propto \mathcal{M}_{0} \beta_{0}^{1 / 2}$. This could in principle also lead to an estimate of the initial normalized mass-to-flux ratio $\mu_{0}$, where $\mu_{0}^{2} \simeq \beta_{0}$ (see Kudoh et al. 2007), if the initial Mach number $\mathcal{M}_{0}$ can be estimated. This is potentially important since a direct measurement of the magnetic field strength using the Zeeman effect is difficult (Crutcher 2012). Indirect probes of the magnetic field such as dust polarization (Hoang \& Lazarian 2008), spectroscopic methods (Auddy et al. 2019), and Faraday rotation (Wolleben \& Reich 2004) also have their limitations. An estimate of $\mathcal{M}_{0}$ can in principle be made from the observed width $\sigma$ of the lognormal PDF by developing an analytic/empirical relation that captures the increase of $\sigma$ with the increasing strength of $\mathcal{M}_{0}^{2} \beta_{0}$ (see Table 1). Such an analysis can be pursued in future work.

\section{CONCLUSION}

Our key findings are:

- The transitional column density $\Sigma_{\mathrm{TP}}$ represents a transition from a turbulent magneticallydominated background ( $\left.\Sigma<\Sigma_{\mathrm{TP}}\right)$ having lognormal shape to a dense region $\left(\Sigma>\Sigma_{\mathrm{TP}}\right)$ with a power-law tail where gravity is dominant.

- $\Sigma_{\mathrm{TP}}$ marks the boundary between regions with subcritical (magnetically dominated) and supercritical (weak magnetic field) mass-to-flux ratio in a star-forming molecular cloud.

- $\Sigma_{\mathrm{TP}}$ depends on the initial velocity perturbation (sonic Mach number $\mathcal{M}_{0}$ ) and the magnetic field strength (plasma $\beta_{0}$ ). Alternatively, it is a measure of the initial Alfvénic Mach number and increases with the increasing strength of $2 \mathcal{M}_{\mathrm{A} 0}^{2}$.

\section{ACKNOWLEDGEMENTS}

SA acknowledges support from an ASIAA Postdoctoral Fellowship. Computations were carried out using facilities of SHARCNET. SB is supported by a Discovery Grant from NSERC.

\section{REFERENCES}

Alves, J., Lombardi, M., \& Lada, C. J. 2014, A\&A, 565, A18

-. 2017, A\&A, 606, L2

Auddy, S., Basu, S., \& Kudoh, T. 2016, ApJ, 831, 46

—. 2018, MNRAS, 474, 400

Auddy, S., Myers, P. C., Basu, S., et al. 2019, ApJ, 872, 207

Ballesteros-Paredes, J., Vázquez-Semadeni, E., Gazol, A., et al. 2011, MNRAS, 416, 1436

Basu, S., Gil, M., \& Auddy, S. 2015, MNRAS, 449, 2413

Burkhart, B. 2018, ApJ, 863, 118

Burkhart, B., Collins, D. C., \& Lazarian, A. 2015a, ApJ, 808, 48

Burkhart, B., Lee, M.-Y., Murray, C. E., \& Stanimirović, S. 2015b, ApJL, 811, L28

Burkhart, B., Stalpes, K., \& Collins, D. C. 2017, ApJL, 834, L1

Blitz, L. 1991, NATO Advanced Science Institutes (ASI) Series C, 3

Collins, D. C., Kritsuk, A. G., Padoan, P., et al. 2012, ApJ, 750,13
Crutcher, R. M. 2012, ARA\&A, 50, 29

Federrath, C., \& Klessen, R. S. 2013, ApJ, 763, 51

Federrath, C., Klessen, R. S., \& Schmidt, W. 2008, ApJL, 688, L79

Goldsmith, P. F., Heyer, M., Narayanan, G., et al. 2008, ApJ, 680, 428

Hoang, T., \& Lazarian, A. 2008, MNRAS, 388, 117

Imara, N., \& Burkhart, B. 2016, ApJ, 829, 102

Kainulainen, J., Beuther, H., Henning, T., \& Plume, R. 2009, A\&A, 508, L35

Könyves, V., André, P., Men'shchikov, A., et al. 2015, A\&A, 584, A91

Körtgen, B., Federrath, C., \& Banerjee, R. 2019, MNRAS, 482,5233

Kritsuk, A. G., Norman, M. L., \& Wagner, R. 2011, ApJL, 727, L20

Kudoh, T., \& Basu, S. 2008, ApJL, 679, L97

—. 2011, ApJ, 728, 123

—. 2014, ApJ, 794, 127 
Kudoh, T., Basu, S., Ogata, Y., \& Yabe, T. 2007, MNRAS, 380,499

Lombardi, M., Alves, J., \& Lada, C. J. 2015, A\&A, 576, L1

Molina, F. Z., Glover, S. C. O., Federrath, C., \& Klessen, R. S. 2012, MNRAS, 423, 2680

Myers, P. C. 2015, ApJ, 806, 226

Padoan, P., Jones, B. J. T., \& Nordlund, Å. P. 1997, ApJ, 474, 730

Passot, T., \& Vázquez-Semadeni, E. 1998, PhRvE, 58, 4501

Patil, A., Huard, D., \& Fonnesbeck, C. 2010, Journal of Statistical Software, Articles, 35, 1.

https://www.jstatsoft.org/v035/i04

Pokhrel, R., Gutermuth, R., Ali, B., et al. 2016, MNRAS, 461, 22

Scalo, J., Vázquez-Semadeni, E., Chappell, D., \& Passot, T. 1998, ApJ, 504, 835

Schneider, N., André, P., Könyves, V., et al. 2013, ApJL, 766, L17
Schneider, N., Csengeri, T., Klessen, R. S., et al. 2015a, A\&A, 578, A29

Schneider, N., Ossenkopf, V., Csengeri, T., et al. 2015b, A\&A, 575, A79

Schneider, N., Bontemps, S., Motte, F., et al. 2016, A\&A, 587, A74

Spitzer, Jr., L. 1942, ApJ, 95, 329

Tassis, K., Christie, D. A., Urban, A., et al. 2010, MNRAS, 408, 1089

van Dyk, D. A. 2003, Hierarchical models, data augmentation, and Markov chain Monte Carlo, ed. E. D. Feigelson \& G. J. Babu, 41-56

Ward, R. L., Wadsley, J., \& Sills, A. 2014, MNRAS, 445, 1575

Wolleben, M., \& Reich, W. 2004, A\&A, 427, 537 